\title{
STRONGLY REVERSIBLE GROUPS
}

\author{
TAKAYUKI TAMURA
}

\begin{abstract}
Following Thierrin [9], a group $G$ is called strongly reversible if for every $x, y \in G$ there are positive integers $l, m, n$ such that $(x y)^{l}=x^{m} y^{n}=y^{n} x^{m}$. This paper studies the structure of strongly reversible groups.
\end{abstract}

1. Introduction. As a generalization of commutative semigroups, Thierrin [9] introduced strongly reversible semigroups as follows:

A semigroup $S$ is called strongly reversible if for every $x, y \in S$ there are positive integers $l, m, n$ such that

$$
(x y)^{l}=x^{m} y^{n}=y^{n} x^{m} \text {. }
$$

Cherubini and Varisco [3], [4], [5] studied strongly reversible archimedean semigroups: $S$ is a strongly reversible archimedean semigroup if and only if $S$ is an ideal extension of a strongly reversible group by a nil semigroup. Accordingly the following question is raised:

What is the structure of strongly reversible groups?

All abelian groups and all torsion groups (namely periodic groups) are strongly reversible but not all groups are. For example, free groups are not. In this paper the author mainly studies the structure of strongly reversible nontorsion groups from the point of group extensions. Although some problem remains, we give a necessary and sufficient condition for a group which is an extension of a torsion group to be strongly reversible. In particular the direct product of a torsion group and a strongly reversible group is strongly reversible. $E-m$ semigroups were defined by Nordahl [7], and it is known that $E-m$ groups are strongly reversible [6]. We show that strongly reversible (semi)groups satisfying some identities turn out to be $E-m$ (semi)groups for some $m$. Also we give an example of a strongly reversible group which is neither torsion nor $E-m$ for any $m>1$.

2. Preliminaries. In this section we give a few basic results which can be easily proved.

LEMMA 1. The class of all strongly reversible semigroups is closed under subsemigroups and homomorphic images.

Throughout this paper, $Z_{+}$denotes the set of positive integers.

Received by the editors April 20, 1980 and, in revised form, June 30, 1981.

1980 Mathematics Subject Classification. Primary 20E34; Secondary 20M10.

$K e y$ words and phrases. Strongly reversible groups, $E$ - $m$ groups, $E-m$ semigroups. 
LEMMA 2. If $(x y)^{l}=x^{m} y^{n}=y^{n} x^{m}$, then $(x y)^{l k}=x^{m k} y^{m k}=y^{m k} x^{m k}$ for all $k \in$ $Z_{+}$.

Let $S$ be a strongly reversible semigroup. For each ordered pair $(x, y)$ of elements of $S$, we define a set $\mathscr{P}_{S}(x, y)$ of triplets of positive integers as follows:

$$
\mathscr{P}_{S}(x, y)=\left\{(l, m, n):(x y)^{l}=x^{m} y^{n}=y^{n} x^{m}\right\} .
$$
$Z_{+}$.

By Lemma 2, if $(l, m, n) \in \mathscr{P}_{S}(x, y)$ then $(k l, k m, k n) \in \mathscr{P}_{S}(x, y)$ for all $k \in$

Immediately we have

Proposition 3. Let $\left\{S_{\lambda}: \lambda \in \Lambda\right\}$ be a set of strongly reversible semigroups. The direct product $\Pi_{\lambda \in \Lambda} S_{\lambda}$ is strongly reversible if and only if for every $\left\{\left(x_{\lambda}, y_{\lambda}\right) \in S_{\lambda} \times\right.$ $\left.S_{\lambda}: \lambda \in \Lambda\right\}$

$$
\bigcap_{\lambda \in \Lambda} \mathscr{P}_{S_{\lambda}}\left(x_{\lambda}, y_{\lambda}\right) \neq \varnothing
$$

3. Extensions of torsion groups. If $G$ is a group, we define as usual $\mathcal{T}(G)$ to be the set of all elements of finite order. Then $\mathscr{T}(G)$ is closed under the unary operation of inverse and is fixed under conjugation. In general, $\mathcal{T}(G)$ is not closed under multiplication.

LEMMA 4. If $G$ is a strongly reversible group, then $\mathcal{T}(G)$ is a normal subgroup of $G$.

Proof. We need to prove only that $\mathscr{T}(G)$ is closed under multiplication. Let $a, b \in \mathcal{T}(G), a^{r}=b^{s}=e$ for some $r, s \in Z_{+}$. By assumption, $(a b)^{l}=a^{m} b^{n}=$ $b^{n} a^{m}$ for some $l, m, n \in Z_{+}$. Using Lemma $2(a b)^{l r s}=a^{m r s} b^{n r s}=e$, hence $a b \in$ $\mathcal{T}(G)$.

$\mathcal{T}(G)$ is called the torsion subgroup of $G$. By Lemma $1, G / \mathcal{T}(G)$ is a strongly reversible group and we see that $G / \mathcal{T}(G)$ is torsion-free, that is, no element of $G / \mathcal{T}(G)$ is of finite order except the identity element.

In summary

THEOREM 5. A strongly reversible group is a group extension of a torsion group by a torsion-free strongly reversible group.

We are more interested in the converse problem, that is,

When is such a group extension strongly reversible?

In the following theorem we do not assume $H$ is torsion-free. We consider a special case of the problem.

THEOREM 6. Let $T$ be a torsion group and $H$ a strongly reversible group. The direct product of $T$ and $H$ is strongly reversible.

Proof. Let $(x, z) \in T \times T,(y, u) \in H \times H$. By assumption, let $(l, m, n) \in$ $\mathscr{P}_{H}(y, u)$. Since $T$ is torsion, there is $r \in Z_{+}$such that $(x z)^{r}=e, x^{r}=e, z^{r}=e$, hence $(l r, m r, n r) \in \mathscr{P}_{T}(x, z)$. By Lemma 2, $(l, m, n) \in \mathscr{P}_{H}(y, u)$ implies $(l r, m r, n r) \in \mathscr{P}_{H}(y, u)$. Therefore $\mathscr{P}_{T}(x, z) \cap \mathscr{P}_{H}(y, u) \neq \varnothing$. By Proposition 3, $T \times H$ is strongly reversible. 
As a special case the direct product of a torsion group and an abelian group is called a fundamental strongly reversible group.

Proposition 7. A direct sum of fundamental strongly reversible groups is a fundamental strongly reversible group.

Next we consider the general case. Let $G \backslash T=\{x: x \in G, x \notin T\}$.

THEOREM 8. Let $G$ be a group extension of a torsion group $T$. Then $G$ is strongly reversible if and only if, for every $x \in G \backslash T, y \in G$, and $a \in T$, there are $l, m, n \in$ $Z_{+}$such that

$$
(a x y)^{l}=x^{m} y^{n}=y^{n} x^{m} .
$$

Proof. Assume $G$ is strongly reversible. For $a \in T, x, y \in G$, there are $j, p, q, k, s, t \in Z_{+}$such that

$$
(a x y)^{j}=a^{p}(x y)^{q}=(x y)^{q} a^{p}
$$

and

$$
(x y)^{k}=x^{s} y^{t}=y^{t} x^{s} .
$$

Since $a \in T, a^{r}=e$ for some $r \in Z_{+}$. By Lemma 2

$$
(a x y)^{j r k}=a^{p r k}(x y)^{q r k}=\left(x^{s} y^{t}\right)^{q r}=x^{s q r} y^{t q r}=y^{t q r} x^{s q r} .
$$

Hence condition (8.0) holds.

Conversely assume (8.0) holds. We want to show

(8.1) For every $z, u \in G,(z u)^{l}=z^{m} u^{n}=u^{n} z^{m}$ for some $l, m, n \in Z_{+}$.

If both $z$ and $u$ are in $T$, it is obvious. The case where $z \in G \backslash T$ and $u \in G$ is immediately obtained by considering $a=e$ in (8.0); the case where $z \in T$ and $u \in G \backslash T$ is obtained by considering $y=e$ in (8.0), that is, since $a^{r}=e$ for some $r \in Z_{+},(8.0)$ becomes $(a x)^{l}=x^{m}=a^{r} x^{m}=x^{m} a^{r}$. Thus we have shown that (8.1) holds in all cases.

Proposition 9.

(9.1) Condition (8.0) is equivalent to the combination of (9.1.1) and (9.1.2) below.

(9.1.1) For every $a \in T$ and $x, y \in G \backslash T$ there are $l, m, n \in Z_{+}$such that $(a x y)^{l}=x^{m} y^{n}=y^{n} x^{m}$.

(9.1.2) For every $a \in T$ and $x \in G \backslash T$, there are $k, r \in Z_{+}$such that $(a x)^{k}=x^{r}$.

(9.2) If $G \backslash T$ is torsion-free and $x, y \in G \backslash T$ are such that $x y \in T$ then $m=n$ in (9.1.1) and $k=r$ in (9.1.2).

Proof. It is easy to see that (8.0) implies (9.1.1) and (9.1.2). Assume (9.1.1) and (9.1.2). To prove (8.0), consider only the case $a \in T, x \in G \backslash T, y \in T$. Note $x y \in G \backslash T$ and $y^{s}=e$ for some $s \in Z_{+}$. Since $T$ is normal in $G, x y=b x$ for some $b \in T$. By (9.1.2) there are $l, m \in Z_{+}$such that $(a x y)^{l}=(x y)^{m}=(b x)^{m}$. Also there are $k, r \in Z_{+}$such that $(b x)^{k}=x^{r}$, hence $(b x)^{k m}=x^{r m}$. Then $(a x y)^{l k}=$ $(b x)^{k m}=x^{r m}=x^{r m} y^{s}=y^{s} x^{r m}$. Therefore we have proved (9.1).

Assume $H=G / T$ is torsion-free, and $x y \in T$ in (9.1.1). Let $\bar{w}$ denote the element of $G / T$ corresponding to $w \in G$. Since $\bar{y}=\bar{x}^{-1},(a x y)^{l}=x^{m} y^{n}=y^{n} x^{m}$ 
implies $\bar{x}^{m-n}=\bar{e}$; then we have $m=n$, since $G / T$ is torsion-free. Similarly we have $k=r$ in (9.1.2).

REMARK 10. Even if $G / T$ is not torsion-free, the additional requirement that $a x=x a$ for all $a \in T$ and $x \in G \backslash T$, implies that $k=r$ in (9.1.2).

4. $E$ - $m$ semigroups, groups. A (semi)group is called an $E-m$ (semi)group if it satisfies the identity $(x y)^{m}=x^{m} y^{m}$. Alperin [1] called it $m$-abelian.

LEMMA 11 [5]. An E-m group is strongly reversible, and it satisfies the identities $(x y)^{j}=x^{j} y^{j}=y^{j} x^{j}$ where $j=m(m-1)$.

Proof. This is an immediate consequence of Theorem 1.9 of [5]; and also a consequence of Theorem 1 of [1].

We consider strongly reversible semigroups or groups $S$ which satisfy

$$
\bigcap_{(x, y) \in S \times S} \mathcal{P}(x, y) \neq \varnothing .
$$

THEOREM 12. Let $S$ be a semigroup. $S$ satisfies the identities $(x y)^{l}=x^{m} y^{n}=y^{n} x^{m}$ for some fixed $l, m, n \in Z_{+}$if and only if $S$ satisfies the identities $(x y)^{k}=x^{k} y^{k}=$ $y^{k} x^{k}$ for some fixed $k \in Z_{+}$.

Proof. Let $y=x, y=x^{2}$ and $x=y^{2}$ respectively to obtain $x^{2 l}=x^{m+n}, x^{3 l}=$ $x^{m+2 n}=x^{2 m+n}$. Either $l=m=n$ and we are done or else $S$ is periodic and there exists $k \in Z_{+}$such that $x^{k}$ is an idempotent for each $x \in S$. In this case, for all $x, y \in S,(x y)^{k}=(x y)^{k l}=\left(x^{m} y^{n}\right)^{k}=x^{m k} y^{n k}=x^{k} y^{k}$.

A periodic group is said to be of bounded exponent if there is an upper bound for the orders of its elements.

THEOREM 13. Let $G$ be a group. The following are equivalent.

(13.1) $G$ satisfies the identities $(x y)^{l}=x^{m} y^{n}=y^{n} x^{m}$ for some fixed $l, m, n \in Z_{+}$.

(13.2) $G$ is an $E$-n group for some $n \in Z_{+}$.

(13.3) $G$ is a group extension of a group $T$ of bounded exponent by an abelian group $H$ and there exists $l \in Z_{+}$such that

$$
(a x y)^{l}=x^{l} y^{l} \text { for all } a \in T, x, y \in G .
$$

Proof. (13.1) $\Rightarrow(13.2)$. This is done by Theorem 12 .

$(13.2) \Rightarrow(13.1)$. By Lemma 11 .

$(13.2) \Rightarrow(13.3)$. By Lemma $11 G$ satisfies the identities

$$
(x y)^{l}=x^{l} y^{l}=y^{l} x^{l}, \quad \text { where } l=m(m-1) .
$$

Let $H_{l}=\left\{x^{l}: x \in G\right\}$. Then $H_{l}$ is a homomorphic image of $G$, and $H_{l}$ is an abelian subgroup of $G$. Let $T_{l}=\left\{x \in G: x^{l}=e\right\}$ where $e$ is the identity element of $G . T_{l}$ is the kernel of the homomorphism $G \rightarrow H_{l}$ and $T_{l}$ has bounded exponent. From the identities it follows that

$$
(a x y)^{l}=a^{l}(x y)^{l}=x^{l} y^{l}
$$

for $a \in T_{l}, x, y \in G$. 
(13.3) $\Rightarrow(13.2)$. Let $x, y \in G$ and $\bar{x}, \bar{y} \in H_{l}$. Since $H_{l}$ is abelian, $(\bar{x} \bar{y})^{2}=\bar{x}^{2} \bar{y}^{2}$. As elements of $G$,

$$
(x y)^{2}=a x^{2} y^{2} \quad \text { for some } a \in T_{l} .
$$

By assumption, for all $x, y \in G$,

$$
(x y)^{2 l}=\left(a x^{2} y^{2}\right)^{l}=x^{2 l} y^{2 l} .
$$

Hence $G$ is an $E-2 l$ group.

COROllary 14. Assume $G$ satisfies the identities $(x y)^{l}=x^{l} y^{l}=y^{l} x^{l}$. If $T_{l}=$ $\mathcal{T}(G)$ then $G \cong T_{l} \times H_{l}$.

Proof. This follows from the fact that $H_{l}$ is a normal subgroup of $G$ and $T_{l} \cap H_{l}=\{e\}$.

5. Examples and problems. We construct an example of a strongly reversible group which is not an $E-n$ group for any $n>1$.

Let $G_{n}$ be a free $E-n$ group over a set $B,|B|>1[8]$.

Let $m \in Z_{+}$with $m>1$, and let $n_{i}=m^{2^{i-1}}, i=1,2,3 \ldots$

Let $G_{n_{i}}$ be the free $E-n_{i}$ group over $B$. By Lemma 1.5 of [5], $E-n_{i}$ groups are also $E-n_{i+1}$ groups. Define $G$ to be the direct sum of $\left\{G_{n_{i}}: n_{i}=m^{2^{i-1}}, i \in Z_{+}\right\}$.

(1) $G$ is strongly reversible.

Let $x, y \in G$. We can assume that $x$ and $y$ are elements of the direct sum of $G_{n_{1}}$, $G_{n_{2}}, \ldots, G_{n_{k}}$ where $n_{i}=m^{2^{i-1}}(i=1,2, \ldots, k)$. By the above remark, $G_{n_{1}}, \ldots, G_{n_{k}}$ are all $E-n_{k}$ groups, hence their direct sum $G_{n_{1}} \oplus \cdots \oplus G_{n_{k}}$ is also $E$ - $n_{k}$. By Lemma 11, there is an $l \in Z_{+}$such that $(x y)^{l}=x^{l} y^{l}=y^{l} x^{l}$. Therefore $G$ is strongly reversible.

$$
G \text { is not an } E-i \text { group for any } i>1 \text {. }
$$

Suppose $G$ is an $E-i$ group for some $i>1$. There is an $n=m^{j^{j-1}}$ for some $j$ such that $i<n$. Since $G_{n}$ is a homomorphic image of $G, G_{n}$ is an $E-i$ group, hence a free $E-n$ group is a homomorphic image of a free $E-i$ group for $i<n$. This contradicts the result (Theorem 2) of [8]: If $G_{n}$ is a homomorphic image of $G_{m}$ then $m \geqslant n$.

In the light of Theorem 8, we offer the following open problems: Is every group extension of a torsion group by a strongly reversible group strongly reversible?

For the next problem, we note that the class $C$ of all $E-m$ groups, $m \in Z_{+}$, is not closed under infinite direct sum. $C$ is closed under finite direct products (Lemma 4 in [8], due to Y. Kobayashi). Is the class of all strongly reversible groups closed under finite direct products?

For the final problem, we observe that a strongly reversible semigroup is right and left reversible [2]. Hence a cancellative, strongly reversible semigroup can be embedded in a group. Is the group of left quotients of a cancellative, strongly reversible semigroup strongly reversible?

ACKNOWLEDGEMENT. The author expresses thanks to the referee for his useful and kind suggestion. 


\section{REFERENCES}

1. J. L. Alperin, A classification of n-abelian groups, Canad. J. Math. 21 (1969), 1238-1244.

2. A. H. Clifford and G. B. Preston, Algebraic theory of semi-groups, Math. Surveys, no. 7, Amer. Math. Soc., Providence, R. I., 1961, pp. 1-224.

3. A. S. Cherubini and A. Varisco, Sui semigruppi fortemente reversibili archimedei, Istit. Lombardo Accad. Sci. Lett. Rend A 110 (1976), 313-321.

4. __ Sui semigruppi fortemente reversibili separativi, Istit. Lombardo Accad. Sci. Lett. Rend. A 111 (1977), 31-43.

5. __ On strongly reversible semigroups, Semigroup Forum 15 (1978), 281-282.

6. __ Some properties of $E$-m semigroups, Semigroup Forum 17 (1979), 153-161.

7. T. Nordahl, Semigroups satisfying $(x y)^{m}=x^{m} y^{m}$, Semigroup Forum 8 (1974), 332-346.

8. T. Tamura, Free E-m groups and free E-m semigroups, Proc. Amer. Math. Soc.84 (1982), 318-324.

9. G. Thierin, Sur quelques propriétés de certaines classes de demi-groupes, C. R. Acad. Sci. Paris 239 (1954), 1335-1337.

Department of Mathematics, University of California, Davis, California 95616 